\title{
Mejora de la calidad de la energía con sistemas fotovoltaicos en las zonas rurales
}

\author{
Improving the quality of energy with photovoltaic systems in rural zones
}

Mellhorar a qualidade da energia com sistemas fotovoltais em zonas ruras

\author{
Maria Rodríguez-Gámez ${ }^{1}$ \\ Antonio Vázquez-Pérez ${ }^{2}$ \\ Alcira Magdalena Vélez-Quiroz ${ }^{3}$ \\ Wilber Manuel Saltos-Arauz ${ }^{4}$
}

Recibido: marzo de 2018

Aceptado: agosto de 2018

Para citar este artículo: Rodríguez-Gámez, M., Vázquez-Pérez, A., Vélez-Quiroz, A.M., y Saltos- Arauz, W.M. (2018). Mejora de la calidad de la energía con sistemas fotovoltaicos en las zonas rurales. Revista Científica, 33(3), 265-274. Doi: https://doi.org/10.14483/23448350.13104

\section{Resumen}

Se expone un análisis vinculado a una de las alternativas energéticas sostenibles que en la actualidad se está adoptando con éxito a nivel mundial con el fin de lograr el mejoramiento de la calidad del servicio eléctrico, ahorrar recursos naturales, reducir las pérdidas y contribuir en la disminución de las emisiones de $\mathrm{CO}_{2}$ a la atmósfera. Se muestran los resultados de un estudio relacionado con el uso de una innovación tecnológica para mejorar la calidad del servicio eléctrico en una comunidad aislada, mediante la introducción de tecnología fotovoltaica conectada a la red de baja tensión de un grupo de 20 viviendas que fueron estudiadas. Se muestran los resultados del estudio de carga y consumo horario de energía de las viviendas y se despliega una metodología propia para el diseño de la tecnología fotovoltaica conectada a la red de baja tensión de las viviendas estudiadas; este tiene el potencial de evitar el consumo de energía de la red convencional y la posibilidad de mejorar la calidad del servicio eléctrico, al tiempo que se reducen el monto de la factura eléctrica y las pérdidas.

Palabras clave: calidad de la energía, desarrollo sostenible, demanda energética, electrificación rural, fuentes renovables.

\begin{abstract}
The paper presents an analysis linked to one of the sustainable energy alternatives that are currently being adopted with worldwide success, in order to improve the quality of electricity service, save natural resources, reduce losses and contribute to the reduction of $\mathrm{CO}_{2}$ emissions into the atmosphere. The results of a study related to an application of technological innovation to improve the quality of the
\end{abstract}

\footnotetext{
Universidad Técnica de Manabí, Portoviejo, Ecuador. taliangel270557@gmail.com

Universidad Técnica de Manabí, Portoviejo, Ecuador. antoniov5506@gmail.com

Universidad Técnica de Manabí, Portoviejo, Ecuador. maqvelez@utm.edu.ec

Universidad Técnica de Manabí, Portoviejo, Ecuador. wsaltos@utm.edu.ec
} 
electric service in an isolated community are presented, by the introduction of photovoltaic technology connected to the low voltage network of a group of 20 houses that were studied. The results of the study of charge and hourly energy consumption of dwellings are shown and an own methodology for the design of the photovoltaic technology connected to the low voltage grid of the houses studied is shown, with potential to avoid the energy consumption of The conventional network, with the possibility of improving the quality of the electric service, while reducing the amount of the electric bill and reducing losses.

Keywords: electrification rural, energy quality, energy demand, renewable sources, sustainable development.

\section{Resumo}

O trabalho apresenta uma análise ligada a uma das alternativas energéticas sustentáveis que atualmente estão sendo adotadas com sucesso em todo o mundo, para alcançar a melhoria da qualidade do serviço elétrico, economizar recursos naturais, reduzir perdas e contribuir para a redução das emissões de $\mathrm{CO}_{2}$ para a atmosfera. Os resultados de um estudo relacionado a uma aplicação de inovação tecnológica para melhorar a qualidade do serviço elétrico em uma comunidade isolada são mostrados, através da introdução de tecnologia fotovoltaica conectada à rede de baixa tensão de um grupo de 20 casas que foram estudadas. Os resultados do estudo de carga e o consumo de energia por hora das casas são apresentados e uma metodologia é implantada para o projeto da tecnologia fotovoltaica conectada à rede de baixa tensão das casas estudadas, com o potencial de evitar o consumo de energia. a rede convencional, com a possibilidade de melhorar a qualidade do serviço elétrico, reduzindo a quantidade de energia elétrica e reduzindo as perdas.

Palavras-chaves: qualidade de energia, fontes renováveis, desenvolvimento sustentável, eletrificação rural, demanda de energia.

\section{Introducción}

La matriz energética del Ecuador opera, en la actualidad, cambios significativos en su estructura y tiene proyectado transitar hacia una base sustentada en la generación con fuentes renovables de energía, aprovechando el potencial que cuenta el país. Aún existen provincias que se encuentran distantes de las centrales generadoras hidráulicas y que dependen en un nivel considerable de la generación basada en el consumo de petróleo, como sucede en la provincia de Manabí. En esta provincia existe un buen potencial solar y pequeños potenciales hidráulicos y eólicos que pueden ser utilizados para la electrificación en el modo de la generación distribuida en función de potenciar a la red convencional y mejorar los parámetros técnicos del sistema. Con ello se logra reducir las pérdidas y garantizar un servicio eléctrico de calidad, en el cual la extensión de las redes no se justifica ni técnica ni económicamente.

En el Plan Nacional para el Buen Vivir 20132017 (Senplades, 2013) se expresa que se podrán implementar pequeños proyectos de generación de energía con fuentes renovables tales como: la fotovoltaica, la eólica, la biomasa y la hidroelectricidad en zonas cercanas a los consumidores; con esquemas de gestión participativa de los gobiernos autónomos descentralizados, las organizaciones comunitarias y el sector privado. Estos proyectos ponen a disposición energías renovables para usos productivos locales y sistemas interconectados, lo que permite generar empleo local, optimizar el uso de los recursos naturales, diversificar los territorios en la generación de electricidad y disminuir las pérdidas técnicas en la transmisión de electricidad.

El servicio eléctrico desempeña un papel importante en el ámbito social, económico y ambiental (Robledo, 2008). El acceso a servicios de electricidad de calidad representa un elemento clave en la lucha contra la pobreza, la marginación, la insalubridad, el analfabetismo y el bienestar de las personas. Por lo que resulta necesario, para promover la eficiencia en los procesos productivos y la eficacia en el servicio público, crear las condiciones para tener comunidades desarrolladas que eviten la migración en los entornos tradicionales. 
Por otro lado, el suministro de electricidad y otros servicios de energía tienen una incidencia directa en las condiciones ambientales. Esto se evidencia en particular en las zonas aisladas, en las que reviste un efecto preventivo en la preservación del medio y los recursos naturales, especialmente para evitar la deforestación descontrolada.

La calidad de la energía resulta de una atención continua; en años recientes esta atención ha sido de mayor importancia debido al incremento del número de cargas sensibles en los sistemas de distribución, las cuales por sí solas, constituyen una causa de la degradación en la calidad de la energía eléctrica (Campos, 2010).

Otros autores la definen cuando la energía eléctrica es suministrada a los equipos y dispositivos con las características y condiciones adecuadas que les permita mantener su continuidad sin que se afecte su desempeño ni provoque fallas a sus componentes (UPME, 2011).

Otra definición plantea que las perturbaciones eléctricas y calidad de la energía, resulta un tema esencial que ha evolucionado en la última década a escala mundial. Está relacionada con las perturbaciones eléctricas que pueden afectar las condiciones de suministro y ocasionar el mal funcionamiento o daño de equipos y procesos. Por tal razón se requiere un tratamiento integral al problema desde diversos frentes, que comprende entre otros: investigación básica y aplicada; diseño; selección; operación; mantenimiento de equipos; normalización; regulación; programas de medición y evaluación; capacitación del personal; entre otros (Holguín y Gómez, 2010).

Cuatro parámetros pueden servir como referencia para clasificar los disturbios de acuerdo a su impacto en la calidad de la energía (Téllez, 2012): Variaciones de frecuencia; Variaciones de amplitud; Variaciones en la forma de onda de voltaje o corriente y; Desbalanceo entre las fases de un sistema polifásico causado principalmente por la operación de cargas monofásicas desiguales que afectan principalmente a máquinas rotatorias y circuitos rectificadores trifásicos.
Por otro lado se puede afirmar que la eficiencia energética consiste en lograr reducir las potencias y energías demandadas al sistema eléctrico, sin que se afecten las actividades normales que se realizan en edificios, industrias o cualquier proceso de la sociedad, pudiendo reducir los costes técnicos y económicos de la explotación (Serra, 2009).

La fuerte dependencia energética al petróleo tiene una incidencia en el acceso y calidad de la electricidad en los territorios rurales del Ecuador, especialmente en la provincia de Manabí, donde existen problemas de calidad de la energía que actualmente se suministra en las zonas rurales $(\mathrm{Mu}-$ rillo, 2005).

Durante el año 2015 la dirección de la institución trazo la estrategia de desarrollar un proyecto encaminado a mejorar la eficiencia y el ahorro energético en el campus universitario y que al propio tiempo pueda propiciar la disminución de la factura que se paga a la empresa eléctrica.

El proyecto se direccionó a demostrar técnicamente las potencialidades de una microrred construida mediante la introducción de una central fotovoltaica conectada a la red de baja tensión, en el edificio n. ${ }^{\circ} 3$ de profesores a tiempo completo de la UTM, en función de evitar el consumo de una parte de la energía de la red convencional, que pueda reducir el monto de la factura eléctrica, propiciando una mejoría en la calidad el servicio eléctrico y contribuir al ahorro de petróleo, así como la disminución de las emisiones de $\mathrm{CO}_{2}$ a la atmósfera (Salto et al, 2017).

Con la política trazada hacia la concientización, la empresa eléctrica ha considerado lograr un impacto social relevante mediante la oferta de energía a las poblaciones que viven en áreas rurales apartadas de la red, mediante la extensión de la longitud de la línea eléctrica. Sin embargo, en algunos casos la extensión longitudinal de la red y el uso de diversas tecnologías destinadas a garantizar un servicio de calidad no son suficientes para lograr este último objetivo, generando molestias en los usuarios. 
La energía solar fotovoltaica es una fuente de energía que produce electricidad de origen renovable obtenida directamente a partir de la radiación solar (Salamanca-Ávila, 2017). Esta se encuentra presente en toda la provincia de Manabí.

El objetivo del trabajo consiste en evaluar la disponibilidad del potencial de las fuentes renovables de energía que permita mejorar la calidad del servicio eléctrico que hoy se oferta en Playa Prieta. La investigación implicó un proceso de análisis y comprensión de la realidad y los problemas vinculados con las posibilidades de aprovechamiento de los recursos energéticos autóctonos de la zona en función de la solución de sus necesidades y el mejoramiento de las condiciones de vida.

Se realizó un estudio de los requerimientos técnicos que intervienen en la calidad del servicio eléctrico y, mediante trabajo de campo, se definieron los problemas que puedan existir. De forma paralela, se evaluó la disponibilidad de las fuentes renovables para la solución de los problemas, específicamente el potencial solar.

\section{Metodología}

Se utilizó una investigación de tipo descriptiva, por cuanto se obtuvo información acerca de la calidad del servicio eléctrico en la zona estudiada. Se describió la realidad relacionada con sus necesidades y la demanda energética. De la situación inicial se pudo evaluar la posibilidad de resolver los problemas mediante el aprovechamiento de las fuentes renovables de energía, en especial la fotovoltaica, dada la disponibilidad de la radiación solar en la zona estudiada. Se evaluó la aplicación de tecnologías vinculadas a las fuentes renovables de energía y se procedió a verificar su impacto económico, técnico y ambiental.

Se aplicaron encuestas a los pobladores de la zona con el fin de precisar el nivel de aceptación que pueden tener las tecnologías vinculadas con las fuentes renovables, con el objetivo de sensibilizar al personal en función de asumir soluciones sostenibles al tema energético.
Se realizaron simulaciones técnicas con el software PVSyst para el diseño de las instalaciones fotovoltaicas que se proponen.

\section{Resultados}

La muestra fue calculada de una población total de 350 residentes de la comunidad Playa prieta, para lo cual se aplicó la ecuación 1, con el objetivo de obtener información confiable por parte de los usuarios respecto al servicio eléctrico, así como el criterio que poseen los mismos relacionado con las soluciones derivadas del uso de las fuentes renovables de energía como una vía segura de precautelar el ambiente.

$$
n=\frac{(Z)^{2} \cdot(P) \cdot(Q) \cdot(N)}{(Z)^{2} \cdot(P) \cdot(Q)+(n e)^{2}}
$$

Donde:

$\mathrm{n} \rightarrow$ Tamaño de la muestra

$Z \rightarrow$ Nivel de confianza

$\mathrm{e} \rightarrow$ Probabilidad de errores $(0.1)$

$\mathrm{P} \rightarrow$ Probabilidad de ocurrencia (0.5)

$\mathrm{Q} \rightarrow$ Probabilidad de no ocurrencia (0.5)

$\mathrm{N} \rightarrow$ Población o universo

Los resultados de 76 encuestas a los pobladores de la comunidad rural fueron tabulados y cuantificados. Se buscó definir una aproximación respecto al juicio que poseen los usuarios relacionado con la calidad del servicio eléctrico, así como los criterios y aceptación respecto a una solución derivada del uso de las fuentes renovables de energía, especialmente de la tecnología fotovoltaica.

La comunidad Playa Prieta es un poblado rural del cantón Portoviejo, en ella se cuenta con el servicio eléctrico. La investigación permitió definir que, a pesar de los esfuerzos realizados por la empresa (que incluye la construcción de una subestación eléctrica), aún persisten dificultades que afectan la calidad del servicio, pues las 
interrupciones se producen semanalmente con un promedio de dos horas y diariamente se padecen problemas de bajo voltaje durante el horario de máxima demanda.

\section{Estudio de la carga}

Como parte de la investigación, se realizó un estudio de la carga a un grupo representativo de 20 viviendas de la comunidad. Para ello se hizo un inventario de los equipos consumidores y el cálculo del consumo diario de la comunidad rural. En la tabla 1 se expone el resumen del estudio de carga realizado a la muestra seleccionada de la comunidad Playa Prieta.

Para el cálculo de la potencia instalada (W) se consideró el inventario de cada equipo por tipo con su potencia nominal unitaria y la cantidad de dispositivos consumidores de energía que existen y se aplicó la ecuación 2.

$$
P=C e * P n u
$$

Donde:

$\mathrm{P} \rightarrow$ Potencia $(\mathrm{W})$

$\mathrm{Ce} \rightarrow$ Cantidad de equipos $(\mathrm{U})$

$\mathrm{Pnu} \rightarrow$ Potencia nominal unitaria $(\mathrm{W})$

Para definir la estimación de energía a consumir en un día se consideró, además, el cálculo de horas de explotación diaria por cada equipamiento o dispositivo consumidor de electricidad, utilizándo la ecuación 3.

$$
E c=P \cdot \frac{h}{d}
$$

Donde:

Ec $\rightarrow$ Estimado de la energía consumida (Wh/día)

$\mathrm{h} \rightarrow$ Horas de trabajo (X horas)

d $\rightarrow$ Día (un día)

El estudio de carga ha permitido calcular que, como promedio por vivienda, existen unos 20 equipos y dispositivos consumidores de energía instalados, con una potencia instalada promedio por vivienda de $5.9 \mathrm{~kW}$ y un consumo diario total promedio de 18 kWh; de los cuales unos 8 kWh se consumen durante las horas del día (correspondiente al 44\%) y 10 kWh se consumen en horario nocturno (56\%).

\section{Estudio del potencial solar}

Se estudió el potencial solar en el sitio de la instalación para valorar la instalación de un sistema fotovoltaico. En la figura 1 se muestra el mapa del potencial solar diario promedio anual en el cantón Portoviejo, de ahí se obtienen los datos para realizar los cálculos energéticos necesarios para estimar la instalación del sistema.

En la tabla 2 se muestra el cálculo de la radiación solar promedio por meses del año que incide en la comunidad Playa Prieta

Tabla 1. Resumen del estudio de carga a la comunidad.

\begin{tabular}{lrrrrr}
\hline Viviendas censadas & $\begin{array}{c}\text { N. } \\
\text { equipos } \\
(\mathbf{U})\end{array}$ & $\begin{array}{c}\text { Potencia } \\
\text { instalada }\end{array}$ & $\begin{array}{c}\text { Consumo } \\
\text { diurno } \\
\text { (W) }\end{array}$ & $\begin{array}{c}\text { Consumo } \\
\text { nocturno }\end{array}$ & $\begin{array}{c}\text { Consumo } \\
\text { total }\end{array}$ \\
\hline Total & 397 & 117268 & 150272 & 206561 & 356.83 \\
Promedio por vivienda & 20 & 5863.4 & 7513.6 & 10328.05 & 17.84 \\
\hline
\end{tabular}

Fuente: elaboración propia de los autores. 


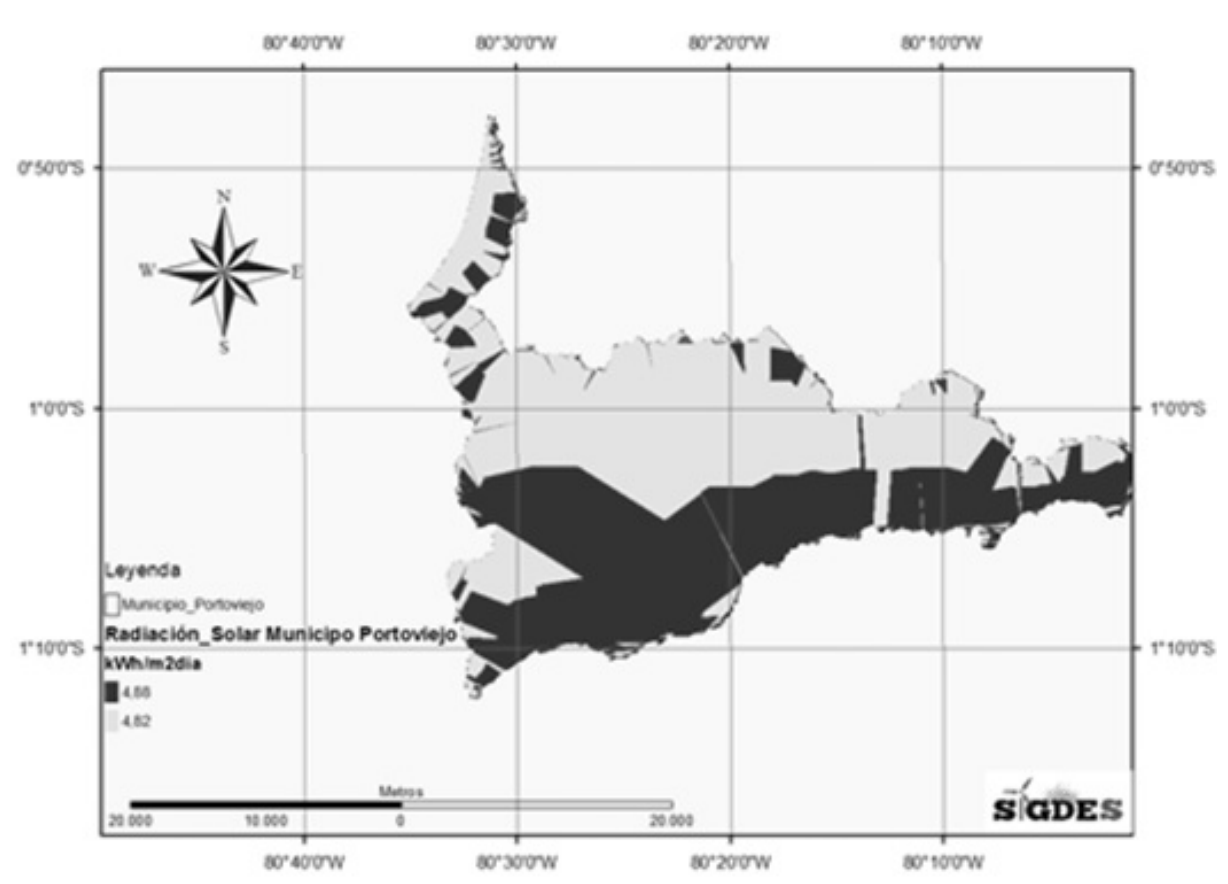

Figura 1. Mapa del potencial solar promedio anual en el municipio Portoviejo.

Fuente: Rodríguez (2015).

Tabla 2. Radiación solar promedio por meses del año.

\begin{tabular}{|c|c|c|c|c|c|c|c|c|c|c|c|c|}
\hline Promedio anual & Ene. & Feb. & Mar. & Abril & Mayo & Junio & Julio & Ago. & Sept & Oct & Nov & Dic \\
\hline 4.845 & 5.399 & 5.275 & 5.856 & 5.769 & 5.231 & 4.086 & 3.802 & 4.096 & 4.420 & 4.458 & 4.607 & 5.149 \\
\hline
\end{tabular}

Fuente: elaboración propia de los autores.

\section{Cálculo de la productividad normalizada (Pn)}

Otro de los datos que resulta necesario calcular es el relacionado con la productividad normalizada, el cual se ha realizado utilizando la ecuación 3.

$$
P n=P s p a \cdot P F V \cdot A c c \cdot \eta t \cdot \eta c
$$

Donde:

$\mathrm{Pn} \rightarrow$ Productividad normalizada (kWh/kWp día) $\mathrm{Pspa} \rightarrow$ Potencial solar promedio anual $(\mathrm{kWh} /$ $\mathrm{m}^{2}$ día)

$\mathrm{PFV} \rightarrow$ Potencia fotovoltaica $(\mathrm{kWp})$
Acc $\rightarrow$ Área de captación solar de las células fotovoltaicas $\left(6.4 \mathrm{~m}^{2}\right)$

$\eta t \rightarrow$ Eficiencia técnica de los módulos (cuando se trata del silicio policristalino es igual al 13\%; si es silicio monocristalino es igual al $16 \%)^{5}$.

Gracias al estudio, se determinó que con la generación fotovoltaica se puede cubrir un $40 \%$ del consumo total de energía en la comunidad rural de Playa Prieta, que corresponde con el gasto energético en horas del día, cuando la radiación solar se encuentra disponible. En el caso de las 20 viviendas estudiadas es equivalente a $150.3 \mathrm{kWh}$ y, conociendo que la productividad normalizada

Sin embargo, en el Ecuador el precio de la energía fotovoltaica es todavía alto, aun cuando el gobierno ha decidido liberar de aranceles a estas tecnologías para su ingreso al país. 
para la comunidad Playa Prieta puede ser igual a $4.316 \mathrm{kWh} / \mathrm{kWp}$ día, se puede calcular la potencia que se requiere instalar en la comunidad para cubrir el consumo de electricidad en horas diurnas y lograr mejorar la calidad del servicio. Para calcular la potencia fotovoltaica que se requiere instalar se utilizó la ecuación 4.

$$
P f v N i=\frac{E C e a}{P n a}
$$

\section{Donde:}

PfvNi $\rightarrow$ Potencia fotovoltaica necesaria a insta$\operatorname{lar}(\mathrm{kWp})$

$\mathrm{ECe} \rightarrow$ Estimado del consumo de electricidad en horario diurno (kWhdía)

Pna $\rightarrow$ Productividad normalizada (kWh/kWp día)

De esta manera se puede estimar que la potencia fotovoltaica que se requiere instalar para cubrir el $40 \%$ del gasto de energía de las viviendas que fueron estudiadas en la comunidad Playa Prieta es de $35 \mathrm{kWp}$.

\section{Posible solución del problema y diseño de la cen- tral fotovoltaica}

Está comprobado que a mayor distancia entre el centro de generación y el usuario se incrementan las pérdidas de energía. Esta situación implica que en determinados momentos la calidad del servicio no sea buena en la comunidad, a pesar de que previamente se ha instalado una subestación.

La posible solución puede radicar en la generación de energía en el modo distribuido mediante el aprovechamiento de las fuentes renovables. En este caso el potencial energético se encuentra con mayor disponibilidad en la zona de estudio, que corresponde al potencial solar disponible los 365 días del año, por lo que se propone la instalación de una central fotovoltaica que pueda cubrir el $40 \%$ de la energía que se consume en las viviendas que fueron estudiadas. Con ello se logra la elevación de la calidad del servicio, al mismo tiempo que se contribuye al ahorro de petróleo y la reducción de las emisiones de $\mathrm{CO}_{2}$ a la atmósfera por concepto de generación de energía eléctrica.

Según el estudio de la carga horaria realizado en la comunidad, y considerando la calidad del potencial solar incidente en la zona, la potencia a instalar es aproximadamente de $35 \mathrm{kWp}$ de tecnología fotovoltaica conectada en el modo de la generación distribuida a la red de baja tensión. Se toma como sitio de instalación las superficies techadas o espacios libres próximos a las viviendas, lo que garantiza que el aporte de energía mejore la calidad del servicio eléctrico y el ahorro de electricidad producida con petróleo.

El diseño de las 20 pequeñas centrales fotovoltaicas que se proponen pueden promediar en la generación diaria por cada pequeña central unos 7.09 kWh, estará integrada técnicamente por un generador fotovoltaico con 10 módulos de silicio policristalino del modelo SF 180 P5-29, con una potencia nominal de $180 \mathrm{Wp}$. El generador estará dispuesto con una cadena en paralelo y 10 módulos en serie, una potencia en condiciones de funcionamiento de $1602 \mathrm{Wp}\left(50^{\circ} \mathrm{C}\right)$, con un $\mathrm{Vmpp}=$ $247 \mathrm{~V}$, Impp $=6.5^{\mathrm{a}}$ y contará con un inversor con una potencia unitaria de $1.5 \mathrm{~kW}$. Una tensión de funcionamiento de $125-400 \mathrm{~V}, 50 / 60 \mathrm{~Hz}$ que posee un grupo de protecciones y un sistema de seguimiento del punto de máxima potencia.

La investigación ha permitido calcular que en un año las 20 viviendas estudiadas pueden consumir un total de 130.2 MWh, de los cuales 54.8 MWh serán en el horario diurno cuando se encuentra disponible la energía solar. Ello supone que si cada pequeña central fotovoltaica que ha sido diseñada puede generar unos 2.59 MWh al año. Entre las 20 instalaciones se estarían generando 51.8 MWh, lo que representa el $40 \%$ del consumo de electricidad total del año de la comunidad. En la figura 2 se observa un esquema del diseño de la central fotovoltaica conectada a la red eléctrica de baja tensión que se propone. 


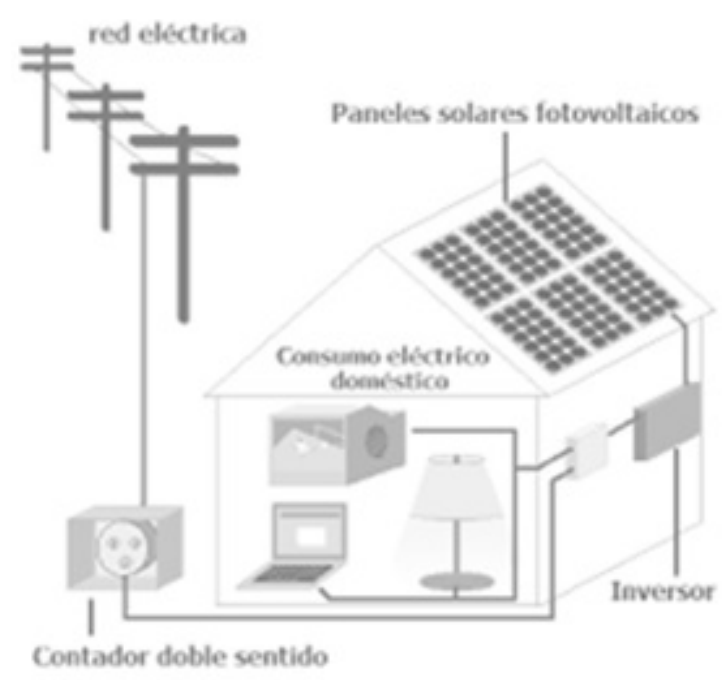

Figura 2. Central fotovoltaica conectada a la red de baja tensión.

Fuente: Sitiosolar 8(2013).

\section{Impactos energético, económico, ambiental y social}

Los estudios de impacto energético, económico, ambiental y social integran los trabajos que deben desarrollarse desde la etapa de planeación del proyecto, pues de sus resultados dependen los elementos que serán tenidos en cuenta para asignar el financiamiento requerido para la inversión.

Desde el punto de vista energético, se pudo determinar que la energía fotovoltaica puede cubrir una parte de la demanda de energía que consume la comunidad, logrando mejorar la calidad del servicio al disminuir las pérdidas técnicas y no técnicas del sistema centralizado convencional. Dada la reducción de petróleo para la generación de electricidad (lo que a su vez disminuye las emisiones de $\mathrm{CO}_{2)}$, se puede apreciar que uno de los logros de la propuesta es la toma de conciencia energética de los pobladores de la comunidad.

La cantidad de energía que puede generar una central fotovoltaica y su destino final se encuentran estrechamente vinculadas con la modalidad y variantes que pueden adoptarse para realizar el análisis de factibilidad económica de una inversión de esa naturaleza.
Con la instalación se reduce el monto de la factura eléctrica en un $40 \%$ para los usuarios de la comunidad rural, logrando un costo adecuadamente competitivo del kWh generado con generación fotovoltaica; además de disminuir la carga económica del Estado en función del subsidio del servicio eléctrico en la provincia.

Los principales efectos ambientales negativos asociados a la introducción de la tecnología fotovoltaica van a estar relacionados con la ocupación del espacio y la intrusión visual al paisaje que se causa con la introducción del equipamiento. La posibilidad de utilizar una parte de la superficie techada de las viviendas reduce de manera importante los efectos de la ocupación del área, pues corresponde a espacios que no se utilizan para otras funciones que no sean las propias de ese tipo de superficies. De modo que la intrusión visual es mínima al encontrarse instalada en la parte superior de las edificaciones.

Al ser instalada en la cubierta de la edificación puede reducir la transferencia de calor hacia el interior, beneficiando la climatización de los locales y con ello el ahorro de energía. Estas características la convierten en una valiosa solución tecnológica que reduce impactos ambientales.

Cuando se consideran las pérdidas asociadas al sistema de generación y suministro energético centralizado, se puede decir que, por cada kWh de energía fotovoltaica suministrada en el modo distribuido de conexión directa a la red de baja tensión de las viviendas, se puede ahorrar más de $1 \mathrm{kWh}$ de electricidad generada con fósiles. Es por ello que puede tener un valor agregado en la reducción de impactos ambientales.

Por otro lado, al considerar el ahorro de petróleo por la generación fotovoltaica y la reducción de las pérdidas, se puede estimar que por cada MWh de electricidad fotovoltaica generada se puede evitar la emisión de 0.9 toneladas de $\mathrm{CO}_{2}$.

En las condiciones que se prevé, la generación fotovoltaica de las 20 centrales fotovoltaicas diseñadas en el modo de la generación distribuida para la comunidad, pueden estar contribuyendo a 
disminuir las emisiones de 47 ton de $\mathrm{CO}_{2}$ a la atmósfera anualmente. Esto durante 25 años, el cual es el ciclo de vida útil de la tecnología.

El impacto social de la puesta en práctica de la tecnología fotovoltaica próximo a los consumidores es positivo ya que estos reciben una mejor calidad de la energía, no lográndose esto a través de las formas tradicionales debido a las pérdidas que se producen en el proceso de trasmisión y distribución. La tecnología fotovoltaica permite, como ninguna otra fuente, la relocalización del recurso energético muy próximo a los consumidores y este puede ser un elemento influyente para la adopción de nuevas posturas de consumo y responsabilidad en función de la preservación de los recursos, así como la adopción de patrones de utilización y gasto energético adecuados a las necesidades reales del trabajo y las personas.

La ubicación de una central fotovoltaica en la superficie techada de las viviendas puede propiciar un cambio importante del rol social de los pobladores de la comunidad, al pasar de ser simples consumidores de energía a poseer la capacidad de generar una parte de la electricidad que consumen mediante el aprovechamiento de un recurso energético limpio, autóctono e inagotable como lo es la energía solar.

La introducción de la tecnología puede convertir a la comunidad rural en pionera en la introducción de las centrales fotovoltaicas conectadas a la red de baja tensión en la provincia, lo que sin dudas contribuye a elevar la imagen ambiental de esta ante la sociedad manabita.

\section{Conclusiones}

La intensidad, calidad y disponibilidad del potencial solar durante todo el año en la comunidad estudiada permite la introducción de la tecnología fotovoltaica para generar energía eléctrica. Esta tiene un rendimiento que es competitivo con cualquier otra de las tecnologías y fuentes de generación existentes, lo que permitirá mejorar la calidad del servicio eléctrico, reducir las pérdidas, disminuir el costo del kWh generado y servido, preservar recursos naturales y disminuir las emisiones de $\mathrm{CO}_{2}$ a la atmósfera.

La instalación de las 20 centrales fotovoltaicas en el modo de la generación distribuida conectadas a la red de baja tensión de las viviendas estudiadas en la comunidad rural de Playa Prieta, permite una mayor relocalización del recurso energético al aprovechar una fuente autóctona, que puede influir en la formación de una nueva conciencia y postura social frente a un consumo más eficiente y el ahorro de energía.

\section{Agradecimientos}

Se agradece a la carrera de Ingeniería Eléctrica de la Facultad de Ciencias, Matemáticas, Físicas y Químicas de la Universidad Técnica de Manabí por el apoyo prestado a la realización del proyecto de investigación en el área rural.

\section{Referencias}

Campos, A. (2010). Calidad de la energía eléctrica. Barranquilla, Colombia: Universidad Autónoma de Occidente. Recuperado de http://www. si3ea.gov.co/Portals/0/Gie/Docs/calidad.pdf

Holguín, M. y Gómez, D. (2010). Análisis de calidad de energía eléctrica en el nuevo campus de la Universidad Politécnica Salesiana. Universidad Politécnica Salesiana. Sede en Guayaquil. Facultad de Ingeniería Carrera de Ingeniería Eléctrica. http://dspace.ups. edu.ec/bitstream/123456789/2110/13/UPSGT000145.pdf.

Murillo, P. (2005). Estudio sobre el servicio de energía eléctrica en el Ecuador y su impacto en los consumidores. Quito, Ecuador: Tribuna Ecuatoriana de Consumidores y Usuarios. Recuperado de http://www.imaginar.org/docs/L tribuna electrico.pdf

Robledo, M. (2008). Calidad de la energía eléctrica: camino a la normalización. En Simposio de Metrología. Santiago de Querétaro, México: 
Comisión Federal de Electricidad. Recuperado de https://www.cenam.mx/simposio2008/sm

Rodríguez, G. (2015). Sistema de Información Geográfica para el Desarrollo Sostenible (Sigdes). Facultad de Ciencias Matemáticas, Físicas y Químicas de la Universidad Técnica de Manabí.

Salamanca-Ávila, S. (2017). Propuesta de diseño de un sistema de energía solar fotovoltaica. Caso de aplicación en la ciudad de Bogotá. Revista Científica, 30(3), 263-277. DOI: https://doi.org/10.14483/23448350.12213

Arauz, W. M. S., Cedeño, G. I., Chávez, S. S., Pérez, A. V. y Gámez, M. R. (2017). Microgrid With a $3.4 \mathrm{kWp}$ Photovoltaic System in the Universidad Técnica de Manabí. International Journal of Physical Sciences and Engineering, 1(2), 11-20. DOI: http://dx.doi.org/10.21744/ ijpse.v1i2.34

Secretaría Nacional de Planificación y Desarrollo (Senplades) (2013). Plan Nacional del Buen Vivir 2013-2017. Quito, Ecuador: Senplades.
Recuperado de http://documentos.senplades. gob.ec

Serra, J. (2009). Guía técnica de eficiencia energética eléctrica. Circutor S.A. Recuperado de http://circutor.com/docs/GUIA EEE SP-LR.pdf

Sitiosolar (2013). El autoconsumo fotovoltaico inyectado a red. Componentes de los sistemas de autoconsumo fotovoltaico inyectado a red. Disponible en: http://www.sitiosolar.com/ el-autoconsumo-fotovoltaico-inyectado-a-red/

Téllez, E. (2012). Calidad de la energía. Programa de ahorro de energía. Puebla. México: Automatización Productiva y Calidad S.A. Recuperado de http://www.watergymex.org

Unidad de Planeación Minero Energética de Colombia (UPME) (2011). Calidad de la energía eléctrica. Bogotá, Colombia: UPME, Instituto Colombiano para el Desarrollo de la Ciencia y la Tecnología, Universidad Distrital Francisco José de Caldas. Recuperado de http://www. andi.hn/wp-content/uploads/2014/11/3-Calidad-de-la-Energía 